\title{
The diagnostic value of PI-RADS V1 and V2 using multiparametric MRI in transition zone prostate clinical cancer
}

\author{
XIMING WANG $^{1 *}$, JIE BAO $^{1 *}$, XIAOXIA PING ${ }^{1}$, CHUNHONG HU $^{1}$, \\ JIANQUAN HOU ${ }^{2}$, FENGLIN DONG ${ }^{3}$ and LINGCHUAN GUO ${ }^{4}$ \\ Departments of ${ }^{1}$ Radiology, ${ }^{2}$ Urology, ${ }^{3}$ Ultrasound and ${ }^{4}$ Pathology, \\ The First Affiliated Hospital of Soochow University, Suzhou, Jiangsu 215006, P.R. China
}

Received November 10, 2017; Accepted May 10, 2018

DOI: $10.3892 / \mathrm{ol} .2018 .9038$

\begin{abstract}
The present study aimed to evaluate the efficacy of using the prostate imaging reporting and data system (PI-RADS) for the detection of prostate cancer (PCa) in the transitional zone (TZ) by 3T multiparametric magnetic resonance imaging (mpMRI), and to compare the diagnostic performance of PI-RADS V1 to V2 for the detection of PCa in the TZ. A total of 77 patients with suspicious lesions in the prostate TZ (83 cores) identified from mpMRI images acquired at $3 \mathrm{~T}$ were scored per the PI-RADS system (V1 and V2) criteria. Magnetic resonance/transrectal ultrasound fusion-guided biopsy was performed in patients with at least one lesion classified as category $\geq 3$ in the PI-RADS V1 assessment. The diagnostic performance of PI-RADS V1 for the detection of $\mathrm{PCa}$ in the TZ was compared with PI-RADS V2 by assessing the sensitivity, specificity and receiver operating characteristics. A total of 31 cases of $\mathrm{PCa}$ in the $\mathrm{TZ}$ and 46 cases of benign prostatic hyperplasia were confirmed by pathology, including 23 cases classified as Gleason score $\geq 7$ and 54 cases of negative results and Gleason score 6. PI-RADS V2 exhibited a higher area under the curve (AUC, 0.888) compared with V1 (AUC,
\end{abstract}

Correspondence to: Professor Chunhong Hu, Department of Radiology, The First Affiliated Hospital of Soochow University, 188 Shizi Road, Suzhou, Jiangsu 215006, P.R. China

E-mail: sdhuchunhong@sina.com

*Contributed equally

Abbreviations: ADC, apparent diffusion coefficient; AUC, area under the curve; DCE, dynamic contrast-enhanced imaging; DWI, diffusion-weighted imaging; MRS, magnetic resonance spectroscopy; MR/TRUS, magnetic resonance/transrectal ultrasound; mpMRI, multiparametric magnetic resonance imaging; $\mathrm{PCa}$, prostate cancer; PI-RADS, prostate imaging reporting and data system; PZ, peripheral zone; ROI, region of interest; T1WI, T1-weighted imaging; T2WI, T2-weighted imaging; TZ, transition zone

Key words: prostate cancer, prostate biopsy, prostate imaging reporting and data system, magnetic resonance/transrectal ultrasound, transition zone, peripheral zone
0.869). The sensitivity of V2 (75.0\%) was higher compared with that of V1 (68.8\%), whereas the specificity of V2 (90.2\%) was lower compared with that of V1 $(96.1 \%)$ at PI-RADS scores of 11 and 4, respectively. The ESUR PI-RADS system may indicate the likelihood of PCa in suspicious lesions in the TZ on mpMRI. These results suggest that PI-RADS V2 performs better compared with $\mathrm{V} 1$ for the assessment of $\mathrm{PCa}$ in the TZ.

\section{Introduction}

Although the majority of cases of prostate cancer (PCa) originate in the peripheral zone (PZ), 25-40\% of tumors arise in the transition zone (TZ) (1). Transrectal ultrasound (TRUS)-guided biopsy is the most frequently used method for diagnosing PCa due to its simplicity, real-time nature and low cost (2). However, conventional TRUS-guided biopsy is limited due to its poor image quality, and low sensitivity and specificity for detecting PCa. The majority of tumors are not visible with the use of sonography, whereby this mode of detection misses 20-30\% of clinically significant cancer cases (3).

Magnetic resonance imaging (MRI) of the prostate is an effective method for the detection of PCa (4). However, tumors in the TZ are difficult to differentiate from other prostate conditions like benign prostatic hyperplasia (BPH) or prostatitis. Presently, multi-parametric MRI (mpMRI) is the most effective method for MR examination of the prostate (4). This method includes protocols for T2-weighted (T2W) high-resolution morphological imaging and functional imaging using diffusion-weighted imaging (DWI), dynamic contrast-enhanced imaging (DCE) and MR spectroscopy (MRS). The combination of mpMRI with magnetic resonance/transrectal ultrasound (MR/TRUS)-targeted biopsy may become a valuable tool for the early detection of clinically significant $\mathrm{PCa}$, while reducing the overdiagnosis of $\mathrm{PCa}$.

The European Society of Urogenital Radiology published a unified scoring system, the 'Prostate Imaging Reporting and Data system (PI-RADS) ', that is based on expert consensus for mpMRI of the prostate, in 2012 (PI-RADS V1) (5) and 2014 (PI-RADS V2) (6). PI-RADS V1 was assigned an individual score for each single MRI sequence, resulting in the determination that a sum of T2WI, DWI and DCE, results in a more valid and reliable method compared with single sequences in predicting the likelihood of PCa on histopathology (6). 
However, the experience with PI-RADS V1 has revealed several limitations of the system.

To address these issues, PI-RADS V2 was developed upon the foundation set by PI-RADS V1. In V1, clinically significant PCa was evaluated by categorizing each single modality (T2WI, DWI, and DCE) on a five-point scale, and the weight of each single sequence in the overall PI-RADS score remained undefined. PI-RADS V2 used the dominant sequences for determining the category of V2 depending on the location of the specific lesion rather than the sum of each component score. DWI and the corresponding apparent diffusion coefficient (ADC) map were used to evaluate the scores from the PZ. T2WI was the dominant sequence noted in the TZ, whereas DCE did not contribute to the overall assessment when the finding had a low (PI-RADS 1 or 2) or high (PI-RADS 4 or 5) likelihood of clinically significant cancer (7). In the PZ region, when the V2 score is 3, a positive DCE may increase the assessment category to a PI-RADS score of 4; likewise, when a TZ lesion has a T2WI score of 3, a DWI score of 5 upgrades the assessment category to a PI-RADS score of 4. As large tumor volumes generally increase the chance of them being clinically significant, a size criterion for T2WI and DWI was proposed to separate a score of 4 from one of 5 in the PZ and TZ regions (8). In addition, a 39-sector scheme was developed in PI-RADS V2, a significant improvement over the 27 PI-RADS V1 sector maps. Finally, the MRS was not included in the PI-RADS V2 score.

The transition zone volume of the prostate gland, comprising two symmetrical lobes, increases with age. Due to its deep location, the prostate is not easily accessed by conventional rectal examination. Despite recent improvements in PI-RADS, the differentiation of $\mathrm{PCa}$ and $\mathrm{BPH}$ remains challenging. $\mathrm{PCa}$ and $\mathrm{BPH}$ manifest as a low signal on T2WI, restrictive diffusion (low ADC) and contrast enhancement (6). The effectiveness of the method for diagnosis of PCa in the TZ is therefore not well established. The purpose of the present study was to compare the diagnostic performance, and predict the risk stratification of PI-RADS V1 and V2 for the diagnosis of PCa in the TZ on mpMRI, based on combined MR/TRUS biopsy.

\section{Materials and methods}

Inclusion criteria. In the present prospectively designed study, consecutive patients who underwent mpMRI at 3.0T between September 2015 and July 2016 were recruited from The First Affiliated Hospital of Soochow University (Suzhou, China). All the patients were referred for prostate MRI from The First Affiliated Hospital of Soochow University by the Department of Urology and presented with clinical suspicion of PCa. Each patient underwent a digital rectal examination and prostate-specific antigen (PSA) test. Inclusion criteria for biopsy included suspicious tumors with at least one lesion with a PI-RADS V1 assessment category of $\geq 3$ (9). A cancer was considered as having originated from a histological zone if $>70 \%$ of its surface was located in the TZ (10).

The following criteria were necessary for inclusion in the present study: No prior treatments received, including radiation therapy or adjuvant hormone therapy; at least one lesion with a PI-RADS V1 assessment category of $\geq 3$; and capacity to successfully undergo 3T MRI with T2WI, DWI and DCE.
Imaging technique. All patients were scanned with a 3T MR scanner (MAGNETOM Skyra; Siemens Healthineers, Erlangen, Germany). The standard spine array coil and an 18-channel body array coil were used for signal reception. The images obtained included transverse T1-weighted turbo spin-echo (TSE) images (repetition time (msec)/echo time (msec), 700/14; section thickness, $3 \mathrm{~mm}$; intersection gap, $0.5 \mathrm{~mm}$; field of view, $25 \mathrm{~cm}$; matrix, 384x336) and transverse, coronal and sagittal T2-weighted TSE images (repetition time (msec) /effective echo time (msec) 6,000/124; section thickness, $3 \mathrm{~mm}$; intersection gap, $0.3 \mathrm{~mm}$; field of view, $25 \mathrm{~cm}$; matrix, 384x336) of the prostate and seminal vesicles. DWI was obtained to calculate an apparent diffusion coefficient using a 2D echo planar imaging sequence with multiple b-value acquisitions $(0,100,800,1,000$ and $1,500 \mathrm{~s} / \mathrm{mm}^{2}$ ), with the diffusion-sensitizing gradients applied along the $\mathrm{X}, \mathrm{Y}$ and $\mathrm{Z}$ axes.

DCE was obtained with 3D T1-weighted gradient-echo volumetric interpolated breath-hold examination in the same plane as the 3D T2W sequence. An intravenous contrast agent (Medtron AG, Saarbruecken, Germany) was administered at $0.1 \mathrm{mmol} / \mathrm{kg}$ body weight at an injection rate of $2.5 \mathrm{ml} / \mathrm{sec}$. Perfusion curves were generated with MR Tissue 4D commercial software (Syngo. via VA20B; Siemens Healthineers) on a Siemens workstation.

Image analysis. An experienced radiologist (with eight years of experience in diagnosing $\mathrm{PCa}$ ) scored the predefined lesion in each of the sequences (T2, DWI and DCE) per PI-RADS V1 and V2 criteria. A second radiologist (with two years of experience in diagnosing $\mathrm{PCa}$ ) separately scored the same region of interest (ROI) using the same method (PI-RADS V1 and V2). Suspicious lesions that were classified as PI-RADS V1 score $\geq 3$ were marked as an ROI, ensuring that the two radiologists were evaluating the same lesions (7). To minimize reporting scoring bias, the mpMRI images were archived for 3 weeks prior to scoring with PI-RADS. A PI-RADS V1 sum score of all three sequences was calculated. The two radiologists had no knowledge of the clinical stage or histopathology of patients and had received prior training with a reference case set for the system (10). Values were recorded as the mean \pm standard deviation.

MR/TRUS fusion-guided biopsy. The patients received intravenous anesthesia prior to undergoing transperineal prostate biopsy. To plan the biopsy puncture, DICOM data of the mpMRI images were guided into a real-time virtual sonography ultrasound host that selected and scored any abnormal signal on the T2WI, DWI or DCE images, and marked the target lesions as the ROI. Sagittal prostate TRUS scans were used to match the ROI and the same section using the urethra, prostate, ejaculatory duct cyst(s) and other anatomical landmarks prior to switching to the axial cross-section using the seminal vesicle, bladder and other anatomical landmarks to further correct the MRI-TRUS images synchronously. Following confirmation of the MRI-TRUS images and TRUS for the prostate sagittal scan, the ' + ' target lesions were identified in real-time. Once partition detail each had been marked, the $3 \mathrm{~mm}$ specimen was fixed $10 \%$ formalin at $37^{\circ} \mathrm{C}$ and sent for pathology puncture analysis.

Statistical analysis. Statistical analyses were performed using SPSS 22.0 (IBM Corp., Armonk, NY, USA). P<0.05 
was considered to indicate a statistically significant difference. Data satisfying the normal distribution assumption were subjected to independent sample t-test. Conversely, data not satisfying the assumption were analyzed using the Mann-Whitney U test. Receiver operating characteristics (ROC) analyses were performed using the sum of scores from the three mpMRI sequences for PI-RADS V1 and V2 using histologically proven biopsy results as the gold standard, and the diagnostic performance of the two scores was repeated using the definition of higher grade PCa having a Gleason score of 7. In addition, optimal thresholds were determined by maximizing the Youden index. The sensitivity, specificity, positive predictive value (PPV), negative predictive value (NPV) and ROC values were calculated, and possible differences between PI-RADS V1 and V2 were evaluated using a nonparametric ROC comparison.

\section{Results}

A total of 77 patients were included in the present study. The mean patient age was $73.20 \pm 6.78$ years and mean PSA level was $26.34 \pm 19.90 \mathrm{ng} / \mathrm{ml}$. A total of 31 cases of $\mathrm{PCa}$ in the TZ and 46 cases of BPH were confirmed by pathology, including 23 cases classified as Gleason $\geq 7$ and 54 cases with negative results plus a Gleason score of 6 . The median volume of the included tumor foci was $2.3 \mathrm{ml}$ (range, 0.5-62.5 ml). The mean age of the group with PCa was greater compared with that of the group with $\mathrm{BPH}(73.20 \pm 6.78$ vs. $71.7 \pm 7.89 ; \mathrm{P}=0.007)$; the mean PSA of the group with $\mathrm{PCa}$ was higher compared with that of the group with BPH $(26.34 \pm 19.90$ vs. $13.98 \pm 13.67$; $\mathrm{P}=0.731$ ), although the difference was not statistically significant (Clinical data are shown in Table I).

All highly suspicious lesions noted on MRI underwent MRI-TRUS fusion puncture (Figs. 1 and 2) within 1 week of the MRI. The PI-RADS V1 and V2 scores were all significantly higher in the cases with PCa compared with those with $\mathrm{BPH}$. The results from the ROC analyses are summarized in Table II, which demonstrated that at the thresholds based on Youden's index, V2 had a higher sensitivity (75.0 vs. 68.8\%) but lower specificity (90.2 vs. 96.1\%) compared with V1 when using the biopsy result as the gold standard. V2 for all lesions exhibited a higher area under the curve (AUC) compared with V1 (0.888 vs. 0.869). Using clinically relevant PCa (Gleason score $\geq 7$ ) as the gold standard, V2 exhibited a higher sensitivity compared with V1 (79.2 vs. 70.8\%) and a lower specificity (83.1 vs. $88.1 \%$ ), whereas the AUC was slightly higher when using V2 compared with V1 (0.86 vs. 0.85). Youden-selected thresholds of 11 for the PI-RADS V1 and 4 for the PI-RADS V2 were determined in the two using the gold standard. The ROC curves are presented in Fig. 3.

\section{Discussion}

The results of the present study demonstrated that for the diagnosis of PCa, the performance of the two PI-RADS versions is different. PI-RADS V2 is a preferable method for the evaluation of PCa in the TZ due to its higher sensitivity (11). As the TZ comprises a mixture of stromal and glandular hyperplasia, $20-30 \%$ of PCa originates in the TZ, and BPH may appear as band-like areas and/or encapsulated round nodules with
Table I. Patient clinical information.

\begin{tabular}{lccc}
\hline & PCa $(\mathrm{n}=31)$ & BPH $(\mathrm{n}=46)$ & P-value \\
\hline Age & $73.2 \pm 6.78$ & $71.7 \pm 7.89$ & 0.007 \\
PSA & $26.34 \pm 19.9$ & $13.98 \pm 13.67$ & 0.731 \\
\hline
\end{tabular}

$\mathrm{BPH}$, benign prostatic hyperplasia; PSA, prostate-specific antigen; $\mathrm{PCa}$, prostate cancer.
A

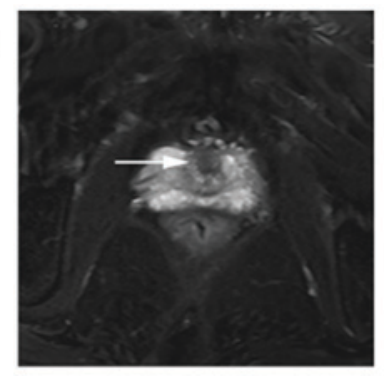

C

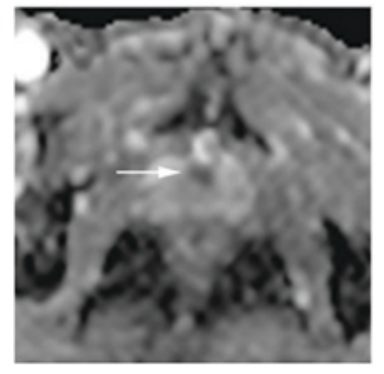

E

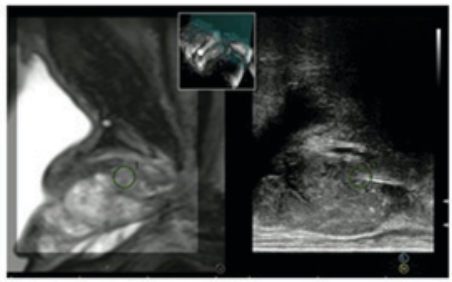

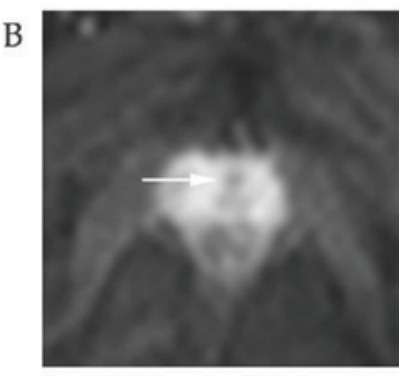
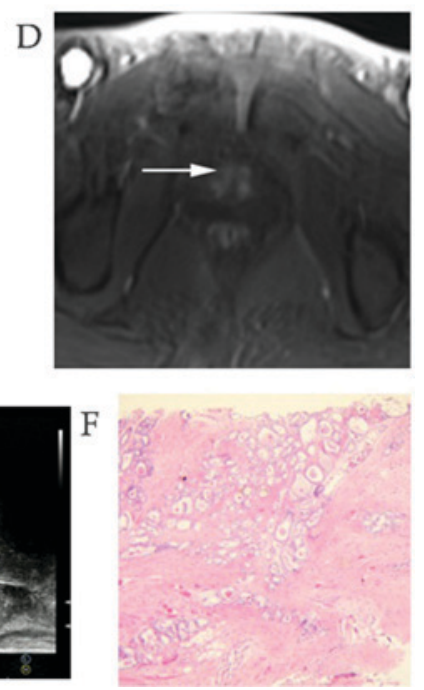

Figure 1. Images of a 71-year-old man who underwent magnetic resonance/transrectal ultrasound fusion-guided biopsy of the prostate TZ. The prostate-specific antigen level was $9.39 \mathrm{ng} / \mathrm{ml}$. (A) The multiparametric magnetic resonance imaging revealed a lesion in the anterior apex of the $\mathrm{TZ}$. T2WI revealed an oval hypointense area in the TZ with a PI-RADS V1 score of 4 and V2 score of 4 . Diffusion-weighted imaging revealed an equal signal intensity lesion on the (B) $1,500 \mathrm{~s} / \mathrm{mm}^{2}$ image and (C) a corresponding low signal intensity lesion on the apparent diffusion coefficient map, resulting in a PI-RADS V1 score of 4 and a V2 score of 3. (D) Dynamic contrast-enhanced imaging revealed early enhancement with a score of 3 in V1 and positive in V2. The sum score of V1 was 11, whereas V2 exhibited a PI-RADS score of 4 (E) The region of interest was located by transrectal ultrasound using T2WI, and real-time needle tracking of the biopsy core is presented in the sagittal section. (F) Histopathology confirmed a Gleason score of $7(3+4)$ for prostate cancer. x200 magnification. PI-RADS, prostate imaging reporting and data system; T2WI, T2-weighted imaging; TZ, transition zone.

circumscribed margins. DWI does not effectively distinguish between PCa and BPH nodules, and the typical DCE patterns of PCa may be absent in the TZ, potentially leading to a decrease in sensitivity using V1 compared with V2 summed scores (9).

In PI-RADS V2, the DCE does not contribute to the overall assessment when the finding has a low (PI-RADS 1 or 2) or 
A

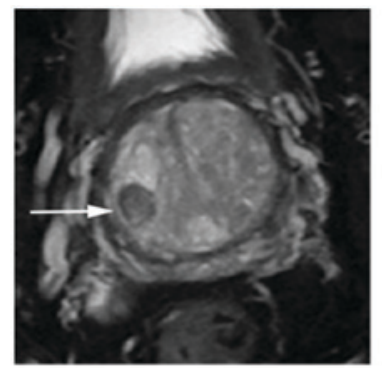

B

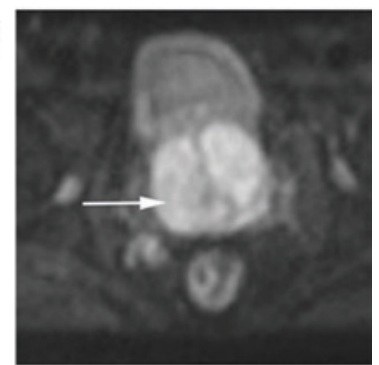

C

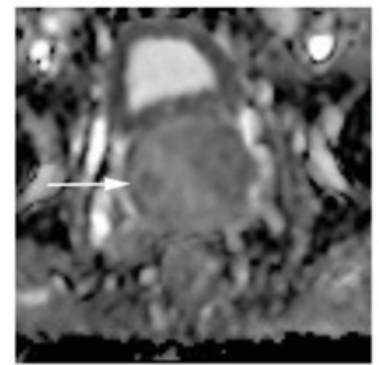

D

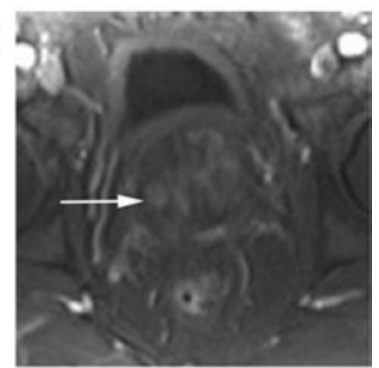

E

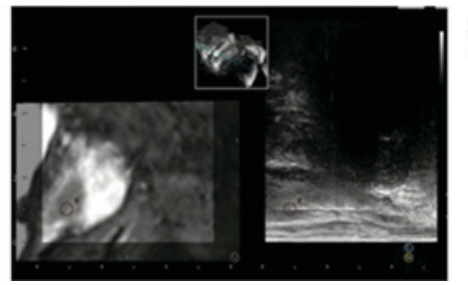

F

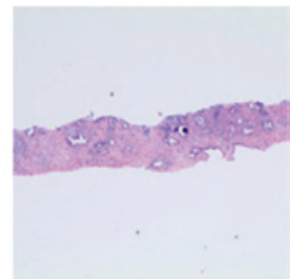

Figure 2. Images of a 78-year-old man with a prostate-specific antigen level of $8.96 \mathrm{ng} / \mathrm{ml}$. (A) T2-weighted imaging revealed an oval hypointense area in the right mid-gland of the transition zone, with a PI-RADS V1 score of 2 and V2 score of 2. (B) Diffusion-weighted imaging had no diffusion restriction on the $15,00 \mathrm{~s} / \mathrm{mm}^{2}$ image; however, (C) apparent diffusion coefficient revealed a slightly hypointense lesion in the corresponding area with a PI-RADS V1 score of 3 and V2 of 2. (D) DCE images revealed an area of early enhancement with a score of 2 in $\mathrm{V} 1$ and a positive DCE magnetic resonance imaging. The sum score of V1 was 7, whereas V2 assigned a PI-RADS score of 2. (E) Real-time needle tracking of the biopsy core is presented in the sagittal section. (F) Histopathology confirmed a benign prostatic hyperplasia lesion. x40 magnification. DCE, dynamic contrast-enhanced imaging; PI-RADS, prostate imaging reporting and data system.

high (PI-RADS 4 or 5) likelihood of identifying clinically significant cancer; if early enhancement on DWI or T2W is suggestive of a cancerous area, DCE is scored as positive. In the absence of enhancement, DCE is scored as negative $(7,11)$. Therefore, PI-RADS V1, which is based on a summed score that assigns the same diagnostic weight to each individual MP-MRI sequence, is limited in its ability to differentiate lesions in the TZ (6).

In the present study, PI-RADS V2 resulted in a significantly higher sensitivity for the diagnosis of lesions in the TZ. Such lesions have always been considered difficult to differentiate on MRI as BPH nodules may exhibit the same restricted diffusion and abnormal enhancement kinetics as $\mathrm{PCa}$ (6). Therefore, DWI and DCE may limit MRI performance in differentiating BPH from PCa and may lead to false-positive findings. In PI-RADS V2, T2W is the primary determining sequence for the TZ, showing the structure of the TZ perfectly, whereas $\mathrm{V} 1$ combines anatomical T2WI with a functional and physiological assessment that includes DWI and its ADC maps, DCE, and other techniques that lead to V2 having a lower specificity.

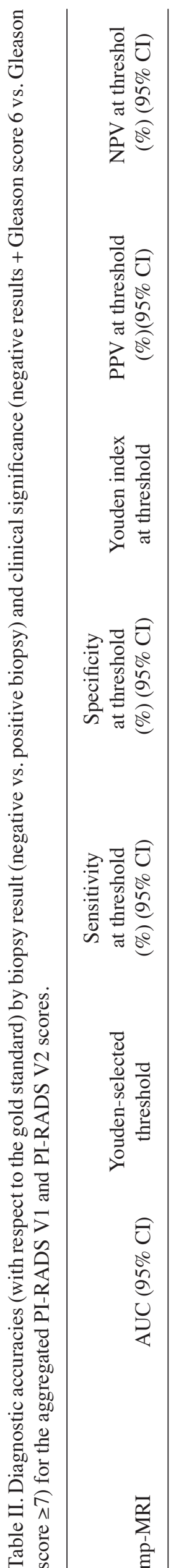

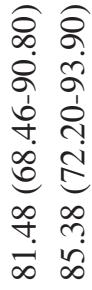

ล트

ते के

ํ.

बi

(1)

$\infty$

สं

导

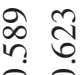

00

‡ั

ลิ

क

는

으 요

๑ 8

하

क 8

t.

$\infty$

$\infty 8$

o in

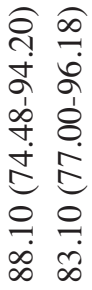

કิ ชิ

एक 8

官

守过

¿ ㄱ

ㅇํㄱ

$=\nabla$

$-$

$\begin{array}{cc}0 & \hat{\alpha} \\ 0 & 0 \\ 0 & 0 \\ 1 & 1 \\ 0 & 0 \\ 0 & 0 \\ 0 & 0 \\ 0 & 0 \\ \infty & 0 \\ 0 & 0\end{array}$

ฮิ

ôे

군

e

ڤ $\infty$

$\stackrel{0}{0}$

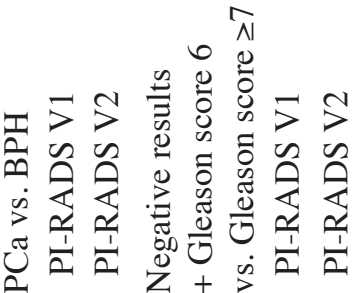



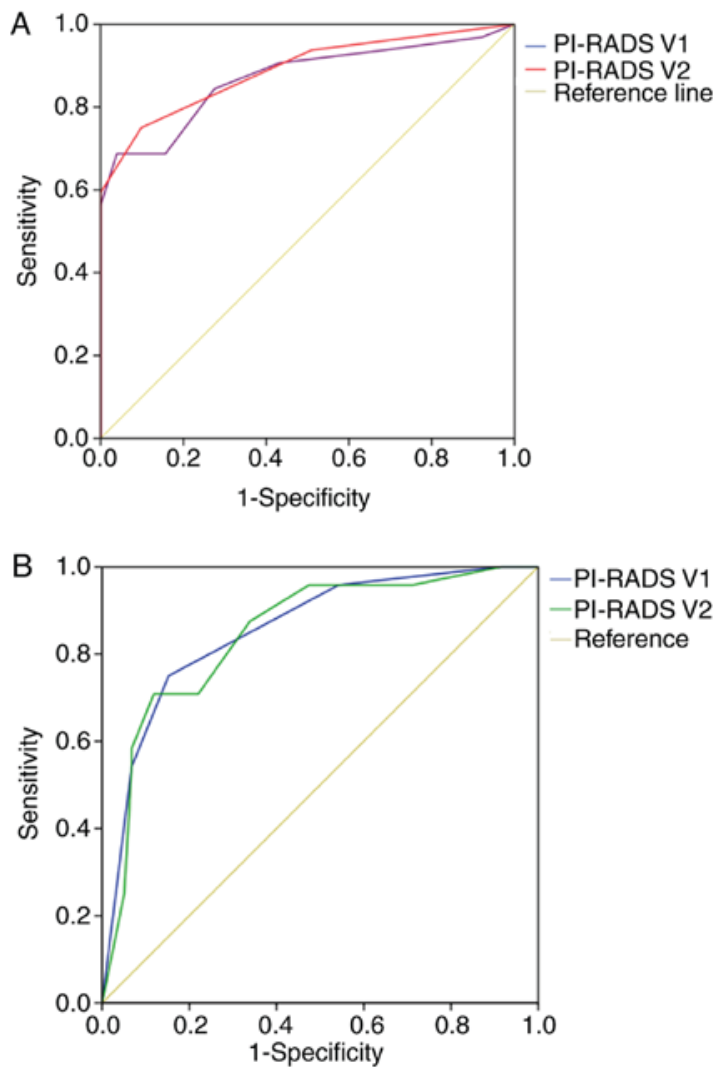

Figure 3. ROC curves. (A) ROC of the biopsy result as the gold standard; and ROC curve presenting the diagnostic performance using PI-RADS V1 and V2 for all lesions in the TZ. The AUCs of PI-RADS V2 were higher compared with those of V1 (AUC 0.87 for V1 and 0.89 for V2). (B) The ROC of the Gleason-based result. The AUCs of V2 were higher compared with those of V1 (AUC 0.85 for $\mathrm{V} 1$ and 0.86 for V2). AUC, area under the curve; PI-RADS, prostate imaging reporting and data system; ROC, receiving operator characteristics curve.

The PI-RADS V2 assessment category for the TZ is determined by the T2WI score, whereas DWI serves that role in the upgrading of uncertain TZ lesions (T2W score of 3) if the DWI score is 5. Subsequently, the AUC of the ROC curves demonstrated an improvement in the diagnostic efficiency of V2 compared with V1. The DCE is reported as positive when there is focal enhancement and no longer features a five-point Likert scale. Its effect in V2 is secondary to T2WI and DWI.

There is little information in the scientific literature suggesting that PI-RADS V2 performs better compared with V1 in the assessment of diagnostic efficiency. In the TZ, according to Polanec (11), there was no significant difference $(\mathrm{P}=0.56)$ in the AUCs between V1 (AUC 0.77 for R1 and 0.78 for R2) and V2 (AUC 0.81 for R1 and 0.84 for R2); the sensitivity was higher for V2 compared with V1, whereas the specificity was lower. Other similar studies have shown that PI-RADS V2 contributes to the risk stratification of PCa with a sensitivity of $77 \%$ and specificity of $68 \%$. These findings are consistent with previous studies: V2 performed better compared with V1 in the detection of $\mathrm{PCa}$ in the $\mathrm{TZ}$ and in the classification for predicting PCa with a lower specificity, whereas V2 maintained excellent sensitivity. One possible reason for the higher diagnostic accuracy of PI-RADS V2 may be structured interpretation criteria. There are no guidelines on how to incorporate the sequence scores that apparently impact the reliability of the reporting (7).
With an increasing emphasis on the diagnostic strategy for detecting clinically significant tumors with PI-RADS, it necessary to predict the risk stratification. Good reliability of the proposed risk stratification using PI-RADS was demonstrated in the present study. A five-point MRI suspicion scale demonstrated excellent risk stratification, with a high sensitivity of $70.8 \%$ and specificity of $88.1 \%$ in PI-RADS V1, and a high sensitivity of $79.2 \%$ and specificity of $83.1 \%$ in PI-RADS V2. However, in V1 and V2, the AUC for the detection of PCa with a Gleason score of $\geq 7$ decreased slightly compared with cancer positivity as the gold standard. The primary reason may be that certain lesions classified as Gleason 6 had lower PI-RADS scores. Larger longitudinal studies are required to further explore this topic.

In a similar study, Roethke et al (6) reported that the sensitivity in PI-RADS V1 was $66.7 \%$ and specificity $91.9 \%$, and Park et al (12) demonstrated that the sensitivity and specificity of PI-RADS V2 for diagnosing PCa with a surgical Gleason score of $\geq 7$ was 84.2 and $58.2 \%$ for Reader 1 and 82.5 and $52.5 \%$ for Reader 2, respectively. As revealed in the present study, a lower specificity was recorded. However, PI-RADS $\mathrm{V} 2$ performed slightly better than $\mathrm{V} 1$ in predicting the risk stratification. As the methodology in other similar studies (e.g., subjects and inclusion criteria) varied, further studies are necessary to provide a fair comparison.

Previous studies have demonstrated that the MR/TRUS fusion-biopsy system is increasingly being used to estimate the diagnostic performance of the PI-RADS scoring system in $\mathrm{PCa}(6,13,14)$. MR/TRUS fusion-biopsy is routinely used in clinical practice to integrate into biopsy planning information gained by MRI (15). MRI-TRUS fusion biopsy has been reported to exhibit a high rate of detection of clinically significant PCa with traditional TRUS biopsy $(2,15,16)$. Currently, TRUS biopsy of the prostate represents the gold standard for diagnosis of PCa prior to surgery; however, the rate of false-negative results may be as high as $35 \%$, depending on the biopsy findings. In addition, the sensitivity and specificity for the detection of malignant lesions are limited. In contrast to traditional TRUS-guided biopsy, MRI-TRUS fusion biopsy effectively avoids the shortcomings of traditional biopsy, using the flexibility of ultrasound biopsy and electronically superimposing this on TRUS images in real time. Furthermore, the present study used transperineal MR/TRUS fusion biopsy. The advantages of a transperineal approach compared with a transrectal one includes reduced susceptibility to compression and mobilization of the prostate (6).

This study had several limitations. First, the primary emphasis was to compare the diagnostic efficacy of PI-RADS V1 and V2 scores without making any conformity assessment between different readers. Second, the study does not compare the diagnostic efficacy of the method for different areas of the prostate. Polanec et al (11) concluded that V2 may be used in the TZ, whereas V1 is still preferable for PZ lesions. Therefore, the area where the lesions are located should be considered when assessing images using the PI-RADS system. Third, the classification used was based on a previous paper; however, further refined classifications are planned for future studies, including an evaluation of Gleason score 6 as an independent group. In addition, there is little research regarding the association between PSA level and PI-RADS system, thus, 
it would be interesting to compare the AUC results on total PSA or percent-free PSA levels in evaluating PCa in TZ.

In conclusion, the present study demonstrated that MRI-TRUS fusion-guided biopsy can be used to efficiently and reliably obtain histological specimens. The PI-RADS scoring system indicated the likelihood of suspicious prostate lesions in the $\mathrm{TZ}$ with high sensitivity and specificity and is useful for risk stratification. These results suggest that PI-RADS V2 performs better compared with V1 for the assessment of PCa in the TZ. Future multi-center clinical trials using PI-RADS V1 and V2 diagnostic scoring should be conducted with multi-center arms and larger patient populations to further expand upon these findings.

\section{Acknowledgements}

The authors would like to thank the Departments of Ultrasound, Urology and Pathology of The First Affiliated Hospital of Soochow University for their valuable help and feedback. The authors would also like to thank Professor Michael E. Mosely, Stanford University, for editing and reviewing the manuscript.

\section{Funding}

No funding was received.

\section{Availability of data and materials}

The datasets used and/or analyzed during the current study are available from the corresponding author on reasonable request.

\section{Authors' contributions}

$\mathrm{CH}$ designed the study. XW and JB contributed equally as first authors. XW and JB performed the literature search and data collection, analyzed the data and interpreted the results, drafted the manuscript, and assisted with the final submitted manuscript. XP, JH and FD participated in the study concept and MRI-TRUS fusion-guided biopsy data acquisition. LG contributed to pathology data analysis and interpretation. All authors read and approved the final manuscript. All authors ensured any questions related to the work were appropriately resolved.

\section{Ethics approval and consent to participate}

Ethics approval and written informed consent were obtained in concordance with the standards of The First Affiliated Hospital of Soochow University Ethics Committee (2016; approval no. 041.

\section{Patient consent for publication}

Study participants provided their consent for the publication of any data and associated images.

\section{Competing interests}

The authors declare that they have no competing interests.

\section{References}

1. Jung SI, Donati OF, Vargas HA, Goldman D, Hricak H and Akin O: Transition zone prostate cancer: Incremental value of diffusion-weighted endorectal MR imaging in tumor detection and assessment of aggressiveness. Radiology 269: 493-503, 2013.

2. Pinto PA, Chung PH, Rastinehad AR, Baccala AA Jr, Kruecker J, Benjamin CJ, Xu S, Yan P, Kadoury S, Chua C, et al: Magnetic resonance imaging/ultrasound fusion guided prostate biopsy improves cancer detection following transrectal ultrasound biopsy and correlates with multiparametric magnetic resonance imaging. J Urol 186: 1281-1285, 2011.

3. Taira AV, Merrick GS, Galbreath RW, Andreini H, Taubenslag W, Curtis R, Butler WM, Adamovich E and Wallner KE: Performance of transperineal template-guided mapping biopsy in detecting prostate cancer in the initial and repeat biopsy setting. Prostate Cancer Prostatic Dis 13: 71-77, 2010.

4. Barentsz JO, Richenberg J, Clements R, Choyke P, Verma S, Villeirs G, Rouviere O, Logager V and Fütterer JJ; European Society of Urogenital Radiology: ESUR prostate MR guidelines 2012. Eur Radiol 22: 746-757, 2012.

5. Hassanzadeh E, Glazer DI, Dunne RM, Fennessy FM, Harisinghani MG and Tempany CM: Prostate imaging reporting and data system version 2 (PI-RADS v2): A pictorial review. Abdom Radiol (NY) 42: 278-289, 2017.

6. Roethke MC, Kuru TH, Schultze S, Tichy D, Kopp-Schneider A, Fenchel M, Schlemmer HP and Hadaschik BA: Evaluation of the ESUR PI-RADS scoring system for multiparametric MRI of the prostate with targeted MR/TRUS fusion-guided biopsy at 3.0 Tesla. Eur Radiol 24: 344-352, 2014.

7. Kasel-Seibert M, Lehmann T, Aschenbach R, Guettler FV, Abubrig M, Grimm MO, Teichgraeber $U$ and Franiel T: Assessment of PI-RADS v2 for the detection of prostate cancer. Eur J Radiol 85: 726-731, 2016.

8. Weinreb JC, Barentsz JO, Choyke PL, Cornud F, Haider MA, Macura KJ, Margolis D, Schnall MD, Shtern F, Tempany CM, et al: PI-RADS prostate imaging-reporting and data system: 2015, version 2. Eur Urol 69: 16-40, 2016.

9. Bouyé S, Potiron E, Puech P, Leroy X, Lemaitre L and Villers A: Transition zone and anterior stromal prostate cancers: Zone of origin and intraprostatic patterns of spread at histopathology. Prostate 69: 105-113, 2009.

10. Röthke M, Blondin D, Schlemmer HP and Franiel T: PI-RADS classification: Structured reporting for MRI of the prostate. Rofo 185: 253-261, 2013 (In German).

11. Polanec S, Helbich TH, Bickel H, Pinker-Domenig K, Georg D, Shariat SF, Aulitzky W, Susani M and Baltzer PA: Head-to-head comparison of PI-RADS v2 and PI-RADS v1. Eur J Radiol 85: 1125-1131, 2016.

12. Park SY, Jung DC, Oh YT, Cho NH, Choi YD, Rha KH, Hong SJ and Han K: Prostate cancer: PI-RADS version 2 helps preoperatively predict clinically significant cancers. Radiology 280 : 108-116, 2016.

13. Lawrence EM, Tang SY, Barrett T, Koo B, Goldman DA, Warren AY, Axell RG, Doble A, Gallagher FA, Gnanapragasam VJ, et al: Prostate cancer: Performance characteristics of combined $\mathrm{T}_{2} \mathrm{~W}$ and DW-MRI scoring in the setting of template transperineal re-biopsy using MR-TRUS fusion. Eur Radiol 24: 1497-1505, 2014.

14. Rastinehad AR, Waingankar N, Turkbey B, Yaskiv O, Sonstegard AM, Fakhoury M, Olsson CA, Siegel DN, Choyke PL, Ben-Levi E and Villani R: Comparison of multiparametric MRI scoring systems and the impact on cancer detection in patients undergoing MR US fusion guided prostate biopsies. PLoS One 10: e0143404, 2015.

15. Roethke M, Anastasiadis AG, Lichy M, Werner M, Wagner P, Kruck S, Claussen CD, Stenzl A, Schlemmer HP and Schilling D: MRI-guided prostate biopsy detects clinically significant cancer: Analysis of a cohort of 100 patients after previous negative TRUS biopsy. World J Urol 30: 213-218, 2012.

16. Xu S, Kruecker J, Turkbey B, Glossop N, Singh AK, Choyke P, Pinto P and Wood BJ: Real-time MRI-TRUS fusion for guidance of targeted prostate biopsies. Comput Aided Surg 13: 255-264, 2008. 